\title{
Different modes of assisted ventilation in patients with acute respiratory failure
}

\author{
D. Chiumello*, P. Pelosi\#, E. Calvi*, L.M. Bigatello", L. Gattinoni*
}

Different modes of assisted ventilation in patients with acute respiratory failure. D. Chiumello, P. Pelosi, E. Calvi, L.M. Bigatello, L. Gattinoni. (C)ERS Journals Ltd 2002.

ABSTRACT: The aim of the present study was to verify that the patient/ventilator interaction is similar, regardless of the mode of assisted mechanical ventilation (i.e. pressure- or volume-limited) used, if tidal volume $\left(V_{T}\right)$ and peak inspiratory flow (PIF) are matched. Therefore, the authors compared the effects of three different modes of assisted ventilation on the work of breathing (WOB) and gas exchange in patients with acute respiratory failure.

For Protocol 1, in seven patients, the authors compared pressure support, assist pressure control and assist control (with square and decelerating wave inspiratory flow pattern) set to deliver the same $V_{T}$ and PIF. For Protocol 2, in another 10 patients, the authors compared pressure support and assist control with high $\left(0.8 \mathrm{~L} \cdot \mathrm{s}^{-1}\right)$ and low $\left(0.6 \mathrm{~L} \cdot \mathrm{s}^{-1}\right)$ PIFs set to deliver the same $V \mathrm{~T}$.

In Protocol 1, there was no difference in WOB and gas exchange between the three modes of assisted ventilation tested. In Protocol 2, the decrease of PIFs during assist control significantly increased WOB.

In conclusion, different modes of assisted ventilation similarly reduce work of breathing and provide adequate gas exchange at fixed tidal volume and peak inspiratory flow only. During assist control, tidal volume and peak inspiratory flow (set by the physician) are the main determinants of the patient/ventilator interaction. Eur Respir J 2002; 20: 925-933.
*Istituto di Anestesia e Rianimazione, Universita' degli Studi di Milano, Ospedale Policlinico-IRCCS, Milano, Italy, "Dipartimento di Scienze Cliniche e Biologiche, Universita' degli Studi dell'Insubria, Varese, Italy, 'Istituto di Anestesia e Rianimazione, Università di Milano-Bicocca, Ospedale S. Gerardo, Monza, Italy.

Correspondence: L. Gattinoni, Istituto di Anestesia e Rianimazione, Ospedale Maggiore Policlinico-IRCCS, via Francesco Sforza, 35, 20122 Milano, Italy. Fax: 390255033230 E-mail: gattinon@polic.cilea.it

Keywords: Acute respiratory failure assist control ventilation assist pressure control ventilation gas exchange

pressure support ventilation work of breathing

Received: September 62001 Accepted after revision: April 212002
A primary goal of mechanical ventilation is to improve gas exchange and reduce the work of breathing (WOB) of patients with acute respiratory failure, without causing iatrogenic lung injury [1]. Assisted ventilation allows the patient to contribute to minute ventilation $\left(V^{\prime} \mathrm{E}\right)$ and offers several advantages over controlled ventilation. It can reduce the need for sedation and paralysis, decrease the risk of barotrauma [2], improve intrapulmonary gas distribution [3], and prevent muscle atrophy $[4,5]$. During assisted ventilation, the WOB is dependent on both the ventilator settings and the patient's ventilatory demand and mechanics.

Pressure support ventilation (PSV) is a pressurelimited, flow-cycled mode of assisted ventilation, in which each breath is supported by a constant level of pressure at the airway $(P$ aw $)$, so that the tidal volume $(V \mathrm{~T})$ and inspiratory flow are more adaptable to the patient's own ventilatory demand [6]. This manner of supporting the patient's own ventilatory effort may be responsible for an improved comfort and synchrony with the ventilator, and has been shown to reduce the WOB and prevent diaphragmatic fatigue in patients with respiratory failure [7]. The main disadvantage of PSV is that it only works best in patients with stable respiratory conditions (i.e. an adequate sufficient ventilatory drive and somewhat preserved respiratory mechanics $[8,9])$.

Assist control ventilation (ACV) is a volume-limited mode of assisted ventilation, in which the $V \mathrm{~T}$ and the peak inspiratory flow (PIF) are set by the clinician and are not altered by the patient's ventilatory demand. If the required $V \mathrm{~T}$ and inspiratory flow are higher than what the ventilator supplies, the WOB increases and the patient may develop fatigue [10]. Consequently, the main advantage of ACV over PSV is that ACV ensures a set $V$ T during conditions of variable ventilatory drive and mechanics [9]. However, several studies have found that during ACV the WOB can increase when the ventilator is unable to meet a patient's high ventilatory demand [11-13]. Comparisons of PSV and ACV, matched to deliver the same $V \mathrm{~T}$, are few and they have only evaluated one $V_{\mathrm{T}}$ in mixed-patient populations (i.e. acute and chronic respiratory failure) $[14,15]$.

Assist pressure control ventilation (APCV) is a pressure-limited mode of assisted ventilation similar to PSV in that the inspiratory flow is delivered at a variable rate and with a decelerating wave flow pattern. It differs from PSV in that the inspiratory 
time is preset, thus providing a greater control of $V \mathrm{~T}$ [1]. A possible advantage of PSV and APCV compared to ACV is the decelerating wave flow pattern, which presents an inspiratory flow rate that is very high at the beginning and variable in a similar manner to a spontaneous breath. Several studies have shown that a decelerating wave flow pattern can reduce the WOB, improve lung mechanics and optimise gas exchange [16-19].

The aim of the present study was to verify that patient/ventilator interaction is similar, regardless of the mode of assisted mechanical ventilation tested, if $V \mathrm{~T}$ and PIF are matched. However, in contrast to previous studies, the authors investigated a homogeneous group of patients, accurately matching $V \mathrm{~T}$ and PIF.

In the first protocol (Protocol 1), the authors compared the effects of different levels of PSV and APCV with the effects of ACV set to deliver equal $V$ T and PIF. ACV was studied with two different wave flow patterns: square and decelerating. In the second protocol (Protocol 2), the effects of PSV and ACV set to deliver a high or low PIF at a constant $V \mathrm{~T}$ were compared.

\section{Materials and methods}

\section{Patients}

The two study protocols were carried out in patients with acute respiratory failure. Patients were included if they were mechanically ventilated with PSV, haemodynamically stable and without evidence of chronic obstructive pulmonary disease (COPD). Seven patients were enrolled in the first protocol and 10 patients were enrolled in the second. The patients' characteristics are reported in table 1, and one patient (patient no. 1) participated in both protocols. The study was approved by the Institutional Review Board and informed consent was obtained from each patient or next of kin.

\section{General protocol}

Three modes of assisted ventilation were compared: PSV, APCV, and ACV [20]. PSV is a pressure-limited, flow-cycled mode of assisted ventilation, in which the ventilator stops applying flow when the inspiratory flow rate decreases below a threshold value; in the authors' ventilator, $30 \%$ of PIF. APCV is a pressurelimited, time-cycled mode of assisted ventilation. ACV is a volume-limited, volume-cycled mode of assisted ventilation.

All patients were mechanically ventilated with a Bearß 1000 mechanical ventilator (Bear Medical Systems Inc., Riverside, CA, USA) [20]. The sensitivity of the pressure trigger was set at $-2 \mathrm{cmH}_{2} \mathrm{O}$. In the APCV and ACV modes, a back-up respiratory rate (RR) of zero was set, so that the RR depended only on the patient's own drive. The inspiratory oxygen fraction $\left(F \mathrm{I}, \mathrm{O}_{2}\right)$ and positive end-expiratory pressure (PEEP) level were previously set by the attending physician on the basis of a PEEP trial [21] and remained constant throughout the study. Patients were allowed to breathe in each modality for $\geqslant 20 \mathrm{~min}$. Respiratory mechanics, arterial blood gas tensions and haemodynamic data were recorded at the end of each study period.

Table 1.-Baseline patients characteristics

\begin{tabular}{|c|c|c|c|c|c|c|c|c|c|}
\hline Patient no. & $\begin{array}{l}\text { Sex } \\
\mathrm{M} / \mathrm{F}\end{array}$ & $\begin{array}{l}\text { Age } \\
\text { yrs }\end{array}$ & $\underset{\mathrm{kg} \cdot \mathrm{m}^{-2}}{\mathrm{BMI}}$ & $\begin{array}{c}\mathrm{PSV} \\
\mathrm{cmH}_{2} \mathrm{O}\end{array}$ & $\begin{array}{l}\text { PEEP } \\
\mathrm{cmH}_{2} \mathrm{O}\end{array}$ & $\begin{array}{c}P \mathrm{~Pa}, \mathrm{O}_{2} / F \mathrm{~F}, \mathrm{O}_{2} \\
\mathrm{mmHg}\end{array}$ & $\begin{array}{c}C_{\text {st, }, \mathrm{rs}} \\
\mathrm{mL} \cdot \mathrm{cmH}_{2} \mathrm{O}^{-1}\end{array}$ & $\begin{array}{c}R \text { tot,rs } \\
\mathrm{cmH}_{2} \mathrm{O} \cdot \mathrm{L}^{-1} \cdot \mathrm{s}\end{array}$ & Diagnosis \\
\hline \multicolumn{10}{|l|}{ Protocol 1} \\
\hline 1 & $\mathrm{~F}$ & 85 & 20.0 & 10 & 7 & 230 & 37.6 & 17.4 & Peritonitis \\
\hline 2 & $\mathrm{~F}$ & 76 & 29.3 & 8 & 4 & 140 & 31.1 & 17.7 & Pneumonia \\
\hline 3 & $\mathrm{~F}$ & 62 & 47.8 & 10 & 9 & 185 & 35.0 & 12.5 & Pneumonia \\
\hline 4 & M & 65 & 27.7 & 20 & 8 & 120 & 27.0 & 10.7 & Peritonitis \\
\hline 5 & M & 19 & 21.6 & 10 & 4 & 310 & 44.3 & 11.5 & Politrauma \\
\hline 6 & M & 60 & 24.7 & 8 & 5 & 328 & 57.9 & 5.1 & Pneumonia \\
\hline 7 & $\mathrm{~F}$ & 40 & 21.5 & 8 & 4 & 377 & 24.4 & 10.4 & Pneumonia \\
\hline \multirow{2}{*}{\multicolumn{10}{|c|}{ Protocol 2}} \\
\hline & & & & & & & & & \\
\hline 1 & $\mathrm{~F}$ & 85 & 20.0 & 10 & 4 & 230 & 37.6 & 17.4 & Peritonitis \\
\hline 2 & $\mathrm{~F}$ & 63 & 47.8 & 18 & 8 & 108 & 32.6 & 8.8 & Pneumonia \\
\hline 3 & $\mathrm{M}$ & 65 & 26.8 & 20 & 10 & 158 & 26.2 & 13.4 & Peritonitis \\
\hline 4 & M & 69 & 21.6 & 6 & 2 & 230 & 60.1 & 15.1 & Pneumonia \\
\hline 5 & M & 80 & 18.5 & 5 & 5 & 373 & 53.3 & 7.2 & Pneumonia \\
\hline 6 & M & 35 & 26.3 & 8 & 7 & 317 & 78.0 & 7.5 & Peritonitis \\
\hline 7 & $\mathrm{~F}$ & 77 & 28.9 & 7 & 4 & 271 & 17.8 & 8.4 & Pneumonia \\
\hline 8 & $\mathrm{M}$ & 61 & 28.7 & 14 & 4 & 210 & 58.0 & 12.2 & Pneumonia \\
\hline 9 & M & 64 & 22.1 & 11 & 6 & 252 & 27.0 & 7.1 & Pneumonia \\
\hline 10 & $\mathrm{~F}$ & 56 & 21.9 & 15 & 6 & 192 & 27.0 & 2.1 & Peritonitis \\
\hline Mean \pm SD & $4 \mathrm{~F} / 6 \mathrm{M}$ & $65 \pm 14$ & $26 \pm 8$ & $11 \pm 5$ & $6 \pm 2$ & $234 \pm 76$ & $44.8 \pm 19.1$ & $9.9 \pm 4.6$ & \\
\hline
\end{tabular}

M: male; F: female; BMI: body mass index; PSV: pressure support ventilation (clinical level used at time of study); PEEP: positive end expiratory pressure; $\mathrm{Pa}_{\mathrm{a}} \mathrm{O}_{2}$ : arterial oxygen pressure; $F \mathrm{I}, \mathrm{O}_{2}$ : inspiratory oxygen fraction; $C$ st,rs: static respiratory system compliance; Rtot,rs: total inspiratory respiratory resistance. 


\section{Protocol 1}

First, one of three levels of PSV $\left(5,15\right.$ and $25 \mathrm{cmH}_{2} \mathrm{O}$ above PEEP) was applied in random order (sealed opaque envelopes). After full data collection, the ventilator was switched to either $\mathrm{ACV}$ with a square wave flow pattern, ACV with a decelerating wave flow pattern, or APCV. ACV was adjusted to provide the same $V$ T and PIF developed at the tested level of PSV. When the wave flow pattern was changed from square to decelerating, the inspiratory time ( $t \mathrm{I})$ automatically increased to maintain a constant $V \mathrm{~T}$. APCV was set at three levels $\left(5,15\right.$, and $25 \mathrm{cmH}_{2} \mathrm{O}$ above PEEP), with a $t$ I equal to the average $t$ I obtained during PSV for the same level of support (fig. 1). These study periods were then repeated in random order at each of the three levels of PSV, so that each patient had performed 12 trials of assisted ventilation by the end of the protocol.

\section{Protocol 2}

The present authors compared PSV to ACV set at two PIF rates, high and low, with both the square and decelerating wave flow patterns. The starting level of PSV was previously chosen by the attending physician on a clinical basis, to obtain an RR of $<40$ breaths per minute $(\mathrm{bpm})$ and a $V \mathrm{~T}$ of $>4-5 \mathrm{~mL} \cdot \mathrm{kg}^{-1}$. After a full baseline data collection, the ventilator was switched to
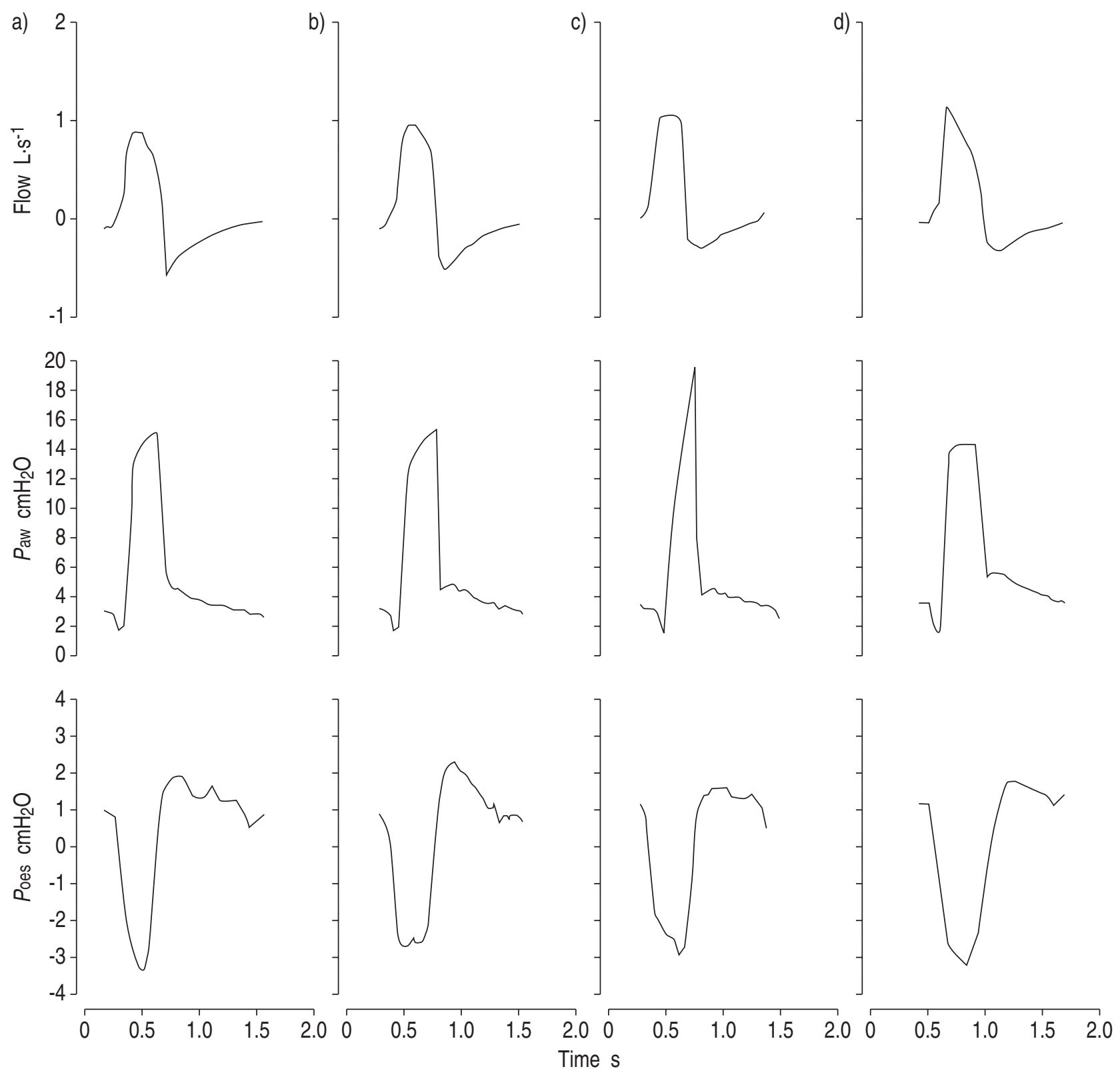

Fig. 1.-An example of airflow, airway $(P$ aw $)$ and oesophageal $\left(P_{\text {oes}}\right)$ pressure tracing during the different modes of assisted ventilation evaluated. a) Pressure support ventilation; b) assist pressure control ventilation; c) assist control ventilation (square wave); d) assist control ventilation (decelerating wave). 
$\mathrm{ACV}$, to deliver an equal $V \mathrm{~T}$ and PIF (high) with each of the two wave flow patterns (square wave high PIF, decelerating wave high PIF, respectively). Using the square wave flow pattern, the PIF was then reduced (low) to reach a $t$ I equal to the average $t$ I previously obtained with the decelerating wave flow pattern (square wave low PIF). The decelerating wave flow pattern was selected, maintaining the constant $V \mathrm{~T}$ and PIF previously selected (decelerating wave low PIF). At the end of protocol, each patient had performed five trials of assisted ventilation.

\section{Measurements}

The following measurements were obtained for each study period. Gas flow rate was measured with a heated pneumotachograph (No. 2; Fleisch, Lausanne, Switzerland) inserted between the proximal tip of the endotracheal tube and the "Y"-piece of the breathing circuit. Paw was measured proximalto the endotracheal tube by a pressure transducer (MPX 2010 DP, Motorola, Phoenix, AR, USA). Oesophageal pressure $(P$ oes $)$ was measured using a radiopaque, thin-walled latex balloon (8-cm long, SmartCath Bicore; Bicore, Irvine, CA, USA), sealed over one end of a polyethylene catheter and connected to a pressure transducer (Bentley Trantec; Bentley Laboratories, Irvine, CA, USA). During measurements, the oesophageal balloon was inflated with $0.5-1.0 \mathrm{~mL}$ of air. Its position and the validity of the pressure signal were assessed by a chest radiograph and by the occlusion test [22]. Both flow and pressure signals were recorded on a polygraph (Brush $2400 \mathrm{~S}$; Gould, Cleveland, OH, USA), processed through an analogue-to-digital converter (100 Hz per channel) and stored on a personal computer for subsequent analysis and computations (Colligo, Elekton, Milan, Italy). The static compliance $(C$ st,rs) and the total inspiratory resistance ( $R$ tot,rs) of the respiratory system were calculated by the inspiratory occlusion method during constant flow inflation (ACV square wave). The $C$ st,rs was computed as the ratio between $V \mathrm{~T}$ and the difference between the endinspiratory and end-expiratory airway pressure. The $R$ tot,rs (including endotracheal tube or tracheostomy resistance) was measured by dividing the difference between the peak $P$ aw and plateau pressure by the inspiratory flow [21].

All patients had an indwelling arterial cannula, connected to a disposable pressure transducer (Transpec IV L974; Abbott Ireland, Sligo, Ireland). Arterial blood gas tensions were measured by a gas analyser (IL-1312 Blood Gas Manager and IL-282 COOximetry; Instrumentation Laboratory, Milan, Italy).

\section{Breathing pattern and respiratory effort}

At the beginning of each data collection period, the authors recorded, for $2 \mathrm{~min}$, consecutive undisturbed breaths to measure PIF, $V \mathrm{~T}, t \mathrm{I}, \mathrm{RR}, V^{\prime} \mathrm{E}$, peak $P$ aw, WOB of the patient per minute $\left(\mathrm{WOB} \cdot \mathrm{min}^{-1}\right.$ ) and per litre $\left(\mathrm{WOB} \cdot \mathrm{L}^{-1}\right)$, and dynamic intrinsic PEEP (PEEPI). VT was obtained by mathematical integration of the flow signal, $V^{\prime} \mathrm{E}$ was calculated as $V \mathrm{~T}$ multiplied by RR, and $t \mathrm{I}$ was the time from the start of inspiration to the point of zero flow between inspiration and expiration. The WOB was computed from a modified Campbell's diagram, where the elastic recoil pressure of the chest wall was taken at the point of sharp inspiratory deflection of the $P$ oes tracings, i.e. the onset of inspiratory effort [23]. The chest wall elastance was calculated at the end of the study by plotting $P_{\text {oes against }} V \mathrm{~T}$ while the respiratory muscles were kept in a state of relaxation by a brief period (3-5 min) of slight hyperventilation to achieve relaxation [24]. PEEPI was considered equal to the differ-

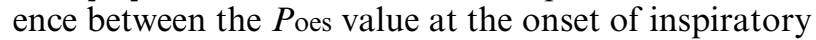
deflection and its value corresponding to the first point at zero flow [25]. Expiratory muscle activity could increase the gastric and oesophageal pressure, and thus overestimate the PEEPI. Therefore, the authors could reasonably exclude such expiratory muscle activity by clinical inspection of the abdomen and chest [23].

In the last $3 \mathrm{~min}$ of each data collection period, three end-expiratory occlusion manoeuvres, $\sim 15 \mathrm{~s}$ apart, were performed using the appropriate knob on the ventilator to measure the mouth occlusion pressure $\left(P_{0.1}\right)$, which estimates the patient's ventilatory drive as the value of $P$ aw at $0.1 \mathrm{~s}$ after the beginning of inspiration [26].

\section{Statistical analysis}

All results are expressed as mean \pm SD. Comparisons between different modes of assisted ventilation were made using one-way repeated measures analysis of variance (ANOVA) and the Bonferroni t-test for multiple comparisons. When the data did not have a normal distribution, a Friedman repeated measures ANOVA as well as a Tukey's test for multiple comparisons were used. A p-value of $<0.05$ was considered statistically significant.

\section{Results}

\section{Protocol 1}

Increasing the level of support during PSV and APCV from 5-25 $\mathrm{cmH}_{2} \mathrm{O}$ caused an increase in $V \mathrm{~T}$ and PIF $(\mathrm{p}<0.05)$ and a decrease in RR $(\mathrm{p}<0.05)$, with no significant change in $V^{\prime} \mathrm{E}$ (table 2). Similarly, the increase of $V \mathrm{~T}$ during ACV caused a decrease in RR $(\mathrm{p}<0.05)$ without significant changes in $V^{\prime} \mathrm{E}$. By increasing the level of $P$ aw or $V$ T from the lowest to the highest studied, the average $\mathrm{WOB} \cdot \mathrm{min}^{-1}$ decreased from $12.2 \pm 3.7 \mathrm{~J} \cdot \mathrm{min}^{-1}$ to $1.4 \pm 0.3 \mathrm{~J} \cdot \mathrm{min}^{-1}(\mathrm{p}<0.05)$, and the average $\mathrm{WOB} \cdot \mathrm{L}^{-1}$ decreased from $0.97 \pm 0.3 \mathrm{~J} \cdot \mathrm{L}^{-1}$ to $0.04 \pm 0.07 \mathrm{~J} \cdot \mathrm{L}^{-1}(\mathrm{p}<0.05)$.

With the exception of the peak $P$ aw, which was higher with ACV with the square wave flow pattern, no differences were found in breathing pattern, WOB, $P 0.1$ and gas exchange when comparing PSV, APCV and ACV matched for the same $V$ T and PIF (table 2).

During ACV, the $t \mathrm{I}$ was lower with the square wave 
DIFFERENT MODES OF ASSISTED VENTILATION

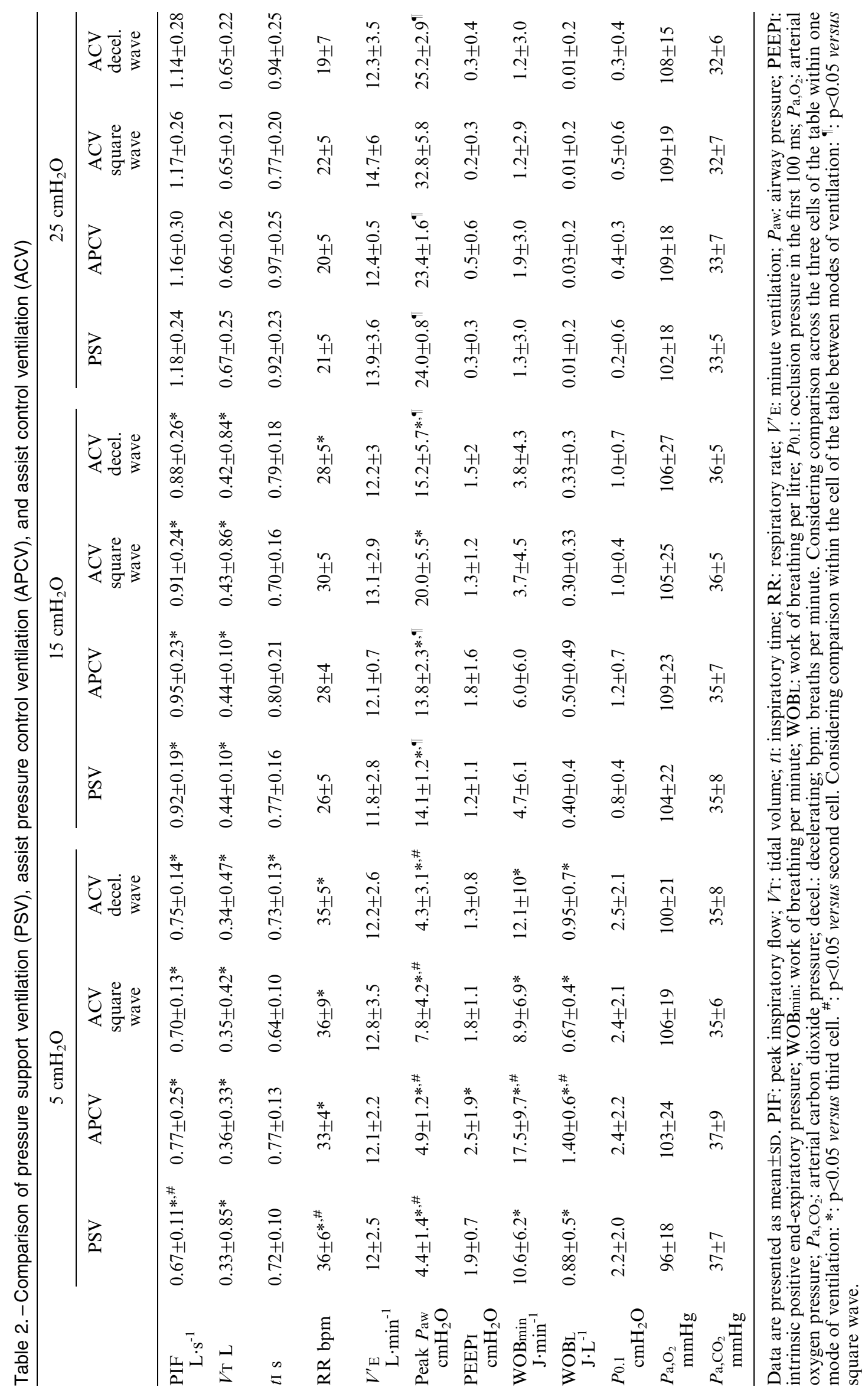


flow pattern compared to the decelerating wave flow pattern, although not significantly. There were no differences in RR, $V^{\prime} \mathrm{E}, \mathrm{WOB}, P_{0.1}$ and gas exchange with the square and the decelerating wave flow patterns matched for the same $V \mathrm{~T}$.

\section{Protocol 2}

The mean level of PSV was set clinically at $11 \pm 5 \mathrm{cmH}_{2} \mathrm{O}$. PSV compared to ACV with the square and decelerating wave flow pattern matched for the same $V \mathrm{~T}$ and PIF, showed no differences in RR, $V^{\prime}$ E, WOB, $P 0.1$ and gas exchange (table 3 ). During ACV with the square wave, the peak $P$ aw was higher than during PSV and ACV with the decelerating square wave flow pattern $(\mathrm{p}<0.05)$. The $t \mathrm{I}$ was significantly lower with the square wave flow pattern than with the decelerating wave flow pattern.

The decrease of PIF maintaining constant $V_{\mathrm{T}}$ caused an increase in WOB $\cdot \mathrm{L}^{-1}$ from $0.29 \pm 0.47 \mathrm{~J} \cdot \mathrm{L}^{-1}$ to $0.40 \pm 0.51 \mathrm{~J} \cdot \mathrm{L}^{-1}$ with the square wave flow pattern $(\mathrm{p}<0.05)$ and from $0.32 \pm 0.43 \mathrm{~J} \cdot \mathrm{L}^{-1}$ to $0.40 \pm 0.50 \mathrm{~J} \cdot \mathrm{L}^{-1}$ with the decelerating wave flow pattern $(p<0.05$, table 3).

\section{Discussion}

The main finding of the present study was that in a uniform patient population recovering from acute respiratory failure, three different modes of assisted ventilation (PSV, APCV and ACV) maintaining a similar $V \mathrm{~T}$ and PIF, had comparable effects on breathing pattern, WOB and gas exchange. With ACV, a lower PIF was associated with an increase in the WOB.
Pressure support ventilation and assist pressure control ventilation

During PSV, the mechanical inflation stops when the inspiratory flow falls below a threshold value. Thus, PSV may adapt to breath-to-breath variability [27] more precisely than APCV, where the mechanical inflation stops after a preset time. However, the present authors found no differences in the patient/ ventilator interaction [28] (e.g. breathing pattern, WOB, gas exchange) with PSV and APCV. Possible explanations are that: 1) breath-to-breath variability of the study's population in terms of RR, $V \mathrm{~T}$ and PIF ( $<10 \%$ during 2 min of recording) was low at each level of selected inspiratory pressure; and 2) by design, the ventilatory settings on PSV and ACV had to be similar, in order to maintain a similar $V \mathrm{~T}$ and PIF.

At high levels of pressure, the cycling criteria of PSV could be a problem in some COPD patients because the inspiratory flow changes more slowly without reaching the threshold value, so these patients start to exhale while the ventilator is still given gas [29]. However, this mainly occurs when the pressure set is excessive, dictating both the breathing pattern and $V \mathrm{~T}$ according to the patient impairment of respiratory mechanics. In these situations, if the physician wants to assist the patient breathing pattern, it would be better to reduce the inspiratory pressure. Conversely, if the physician wants to control the delivered $V \mathrm{~T}$, the APCV seems more appropriate.

\section{Assist control ventilation and pressure support ventilation}

During ACV, all breaths are delivered with a set $V \mathrm{~T}$, PIF and wave flow pattern (square or decelerating). The fast and variable inspiratory flow characteristics of PSV may be more suitable than a fixed flow to match the patient's own ventilatory effort [6]. However,

Table 3. - Comparison of pressure support ventilation (PSV) and assist control ventilation (ACV) with high and low inspiratory peak inspiratory flow (PIF)

\begin{tabular}{|c|c|c|c|c|c|}
\hline & \multirow[t]{2}{*}{ PSV } & \multicolumn{2}{|c|}{ High PIF } & \multicolumn{2}{|c|}{ Low PIF } \\
\hline & & $\begin{array}{c}\text { ACV } \\
\text { square wave }\end{array}$ & $\begin{array}{c}\text { ACV } \\
\text { decel. wave }\end{array}$ & $\begin{array}{c}\text { ACV } \\
\text { square wave }\end{array}$ & $\begin{array}{c}\text { ACV } \\
\text { decel. wave }\end{array}$ \\
\hline $\mathrm{PIF} \mathrm{L} \cdot \mathrm{s}^{-1}$ & $0.78 \pm 0.19$ & $0.80 \pm 0.20$ & $0.78 \pm 0.20^{\bullet}$ & $0.65 \pm 0.19^{*}, \#$ & $0.64 \pm 0.18^{+, \#, *}$ \\
\hline$V \mathrm{~T} \mathrm{~L}$ & $0.37 \pm 0.11$ & $0.38 \pm 0.12$ & $0.37 \pm 0.11$ & $0.38 \pm 0.11$ & $0.37 \pm 0.12$ \\
\hline$t \mathrm{I} \mathrm{s}$ & $0.89 \pm 0.39$ & $0.76 \pm 0.33^{\#}$ & $0.88 \pm 0.35^{*}$ & $0.86 \pm 0.35$ & $1.10 \pm 0.47^{*,+, \boldsymbol{\Phi}, \#}$ \\
\hline RR bpm & $31 \pm 11$ & $31 \pm 10$ & $30 \pm 10$ & $30 \pm 9$ & $29 \pm 9$ \\
\hline$V^{\prime} \mathrm{E} \mathrm{L} \cdot \mathrm{min}^{-1}$ & $10.8 \pm 3.2$ & $11.5 \pm 3.3$ & $10.8 \pm 3.0$ & $11.3 \pm 3.0$ & $9.9 \pm 2.7$ \\
\hline Peak $P$ aw $\mathrm{cmH}_{2} \mathrm{O}$ & $9.9 \pm 5.7 *$ & $17.1 \pm 6.3$ & $10.9 \pm 4.8^{*}$ & $14.7 \pm 6.8$ & $9.3 \pm 3.0 *$ \\
\hline PEEPI $\mathrm{cmH}_{2} \mathrm{O}$ & $1.5 \pm 1.3$ & $1.7 \pm 2.2$ & $1.8 \pm 2.2$ & $1.7 \pm 2.2$ & $2.6 \pm 3.0$ \\
\hline $\mathrm{WOB} \min \mathrm{J} \cdot \mathrm{min}^{-1}$ & $2.9 \pm 3.0$ & $3.3 \pm 5.8$ & $3.5 \pm 5.2$ & $4.1 \pm 6.1 *$ & $3.8 \pm 5.3$ \\
\hline WOBL $\mathrm{J} \cdot \mathrm{L}^{-1}$ & $0.30 \pm 0.28$ & $0.29 \pm 0.47$ & $0.32 \pm 0.43$ & $0.40 \pm 0.51 *$ & $0.40 \pm 0.50^{+}$ \\
\hline$P 0.1 \mathrm{cmH}_{2} \mathrm{O}$ & $1.5 \pm 1.0$ & $1.5 \pm 1.0$ & $1.8 \pm 1.9$ & $1.9 \pm 1.8$ & $1.9 \pm 1.7$ \\
\hline$P \mathrm{a}, \mathrm{O}_{2} \mathrm{mmHg}$ & $97 \pm 16$ & $96 \pm 17$ & $93 \pm 12$ & $99 \pm 21$ & $96 \pm 16$ \\
\hline $\mathrm{Pa}, \mathrm{CO}_{2} \mathrm{mmHg}$ & $39 \pm 6$ & $39 \pm 8$ & $39 \pm 7$ & $40 \pm 9$ & $40 \pm 9$ \\
\hline
\end{tabular}

Data are presented as mean \pm SD. PIF: peak inspiratory flow; $V \mathrm{~T}$ : tidal volume; $t \mathrm{I}$ : inspiratory time; RR: respiratory rate; $V^{\prime} \mathrm{E}$ : minute ventilation; $P$ aw: airway pressure; PEEPI: intrinsic positive end-expiratory pressure; WOBmin: work of breathing per minute; WOBL: work of breathing per litre; $P_{0.1}$ : occlusion pressure in the first $100 \mathrm{~ms} ; P_{\mathrm{a}, \mathrm{O}_{2}}$ : arterial oxygen pressure; $P_{\mathrm{a}}, \mathrm{CO}_{2}$ : arterial carbon dioxide pressure; decel.: decelerating; bpm: breaths per minute. *: $\mathrm{p}<0.05$ versus ACV square wave high PIF; ${ }_{\#}: \mathrm{p}<0.05$ versus PSV; $:$ : $<<0.05$ versus ACV square wave low PIF; ${ }^{+}: \mathrm{p}<0.05$ versus ACV decelerating wave high PIF. 
PSV has potential shortcomings. Hypoventilation may occur in the presence of abnormal respiratory mechanics, (i.e. decreased compliance and/or increased resistance), due to the imbalance between the inspiratory pressure delivered and the high inspiratory workload, even with an adequate respiratory drive.

TEJEDA et al. [14] and KREIT et al. [15] evaluated the effects of ACV and PSV on gas exchange and respiratory mechanics, respectively. As in the present study, these authors did not find significant differences between the two modes of ventilation. These studies, however, only investigated one level of $V \mathrm{~T}$.

The WOB performed by the authors' patients was identical during ACV and PSV (table 2) and was similar to values measured in patients with acute respiratory failure ventilated with ACV [15]. A seminal paper by MARINI et al. [11] showed that substantial work could be generated by patients during ACV when their own inspiratory flow did not match the pattern delivered by the ventilator. In some cases, the patients' WOB during machine-assisted cycles exceeded that of unsupported breaths $[11,12]$.

The present results differ from the observations of MARINI et al. [11] for the following reasons. 1) In the present study, ACV was better matched (using fixed $V \mathrm{~T}$ and PIF) to the patients' ventilatory demands and breathing patterns. 2) MARINI et al. [11] used an older type of mechanical ventilator (i.e. one with a slower trigger phase and inspiratory flow delivery). In the present protocols, the trigger sensitivity was the same with ACV and PSV and the time delay was minimal, $<100 \mathrm{~ms}$. 3) The patient populations studied are different. The present authors' patients were all in a stable phase of acute respiratory failure, with no significant pre-existent respiratory disease. Those studied by MARINI et al. [11] were more heterogeneous, including patients with both acute and chronic respiratory failure, with and without the use of PEEP as part of the ventilatory strategy. Excluding COPD patients from the present study may have prevented increased WOB was caused by abnormality of the patients' respiratory mechanics (e.g. increased airway resistance, PEEPI), rather than or in addition to a characteristic of the ventilator.

The present authors found that ACV with the square wave form was as effective as PSV when the inspiratory time was reduced to obtain the same PIF. However, this is not commonly performed in clinical practice because of the risk of high peak $P$ aw (tables 2 and 3 ) and possible patient/ventilator disynchrony. The authors found that the patient/ventilator interaction was similar, independent of the mode, if $V \mathrm{~T}$ and PIF were matched. However, the PSV may be the easiest to set at the bedside, even when possible risks are into account.

\section{Assist control ventilation and assist pressure control ventilation}

APCV is characterised by a rapid increase of pressure at the airway, with a decelerating wave flow pattern $[19,30]$. In a similar manner to PSV, the resulting $V_{\mathrm{T}}$ is determined by the capability of the ventilator and the patient's respiratory mechanics. As in any pressure-limited mode, the $V \mathrm{~T}$ can be substantially reduced by acute changes in respiratory mechanics, with the consequent risk of hypoventilation, although its clinical significance is not clearly documented. KALLET et al. [31] found a lower WOB with APCV than with volume control ventilation, due to the advantage of the high inspiratory flow pattern of APCV. Cinnella et al. [32] observed a decrease in WOB using APCV compared to ACV only at a moderate $V \mathrm{~T}$ (i.e. $V \mathrm{~T}$ of $470 \mathrm{~mL}$ ) and low inspiratory flow rate. In contrast, no differences in WOB with ACV (either with square or decelerating wave flow pattern) and APCV were found in this study. This discrepancy with the results of CinNella et al. [32] may be due to the different patient population studied, i.e. patients with COPD and air flow limitation, in whom the particular flow pattern of APCV, variable and maximum at the beginning of inspiration, is likely to be beneficial and generate higher $V \mathrm{~T}$. Furthermore, Cinnella et al. [32] did not match the two modes for the same PIF. Assist control ventilation: effect of square and
decelerating wave flow pattern

Traditionally, volume-limited breaths during ACV are delivered with a square flow pattern (i.e. constant flow). In the latest generation of mechanical ventilators, however, the decelerating wave flow pattern is often available even in the volume-limited modes [33]. With the square wave flow pattern, the flow rate remains relatively constant after reaching the PIF, while with the decelerating wave flow pattern, the flow rapidly reaches the PIF value and then decreases linearly afterwards to $50 \%$ by the end of inspiration [20]. In patients with normal respiratory mechanics, different wave flow patterns have no major effect on gas exchange [34]. Also, no differences were found in gas exchange and WOB between the two wave forms. The present results differ with those of AL SAADY and BENNETT [33], in which the decelerating wave flow pattern produced a significant increase in arterial oxygenation. The decelerating wave flow pattern may be more effective in patients with heterogeneous respiratory mechanics and prolonged alveolar time constants (i.e. high compliance and high resistance) characteristic of COPD, or in patients with more severe lung injury [32]. In these circumstances, a high PIF followed by a slow decline may allow more time for gas distribution in the alveoli.

The square wave, which maintained a high flow throughout the inspiratory time, raised peak $P$ aw more than the decelerating wave flow pattern during volume and pressure target ventilation (tables 2 and 3). An excessive PIF and a high peak $P$ aw might cause patient discomfort and patient/ventilator disynchrony [6].

\section{Assist control ventilation: effect of different peak inspiratory flow}

Varying the PIF setting between $0.6-0.8 \mathrm{~L} \cdot \mathrm{s}^{-1}$ had a mild impact on the patients' WOB; with the square 
waveform, the lower PIF was associated with a slightly but significantly higher WOB (table 3). WARD et al. [35] measured the WOB in patients with acute respiratory failure, changing the PIF between $0.4-1.1 \mathrm{~L} \cdot \mathrm{s}^{-1}$, while maintaining a constant $V \mathrm{~T}$. It was demonstrated that at low PIF, the patient's WOB approached $65 \%$ of the total inspiratory work. When the PIF was progressively raised to $0.9 \mathrm{~L} \cdot \mathrm{s}^{-1}$, the patient's WOB was reduced to $10 \%$ of the total inspiratory work. Further increases of PIF $\leqslant 1.1$ did not affect the WOB [5]. In a previous study, similar results were obtained by the present authors, by increasing the PIF during PSV, where the highest PIF did not further reduce the WOB and was associated with patient discomfort [36]. Both these studies suggest that there is a threshold of flow demand below which a lower flow rate increases the WOB and above which a further increase of flow rate is not beneficial and may be uncomfortable $[33,35]$. In the present study, only minor variations of PIFs were applied, according to clinical indications, and the authors did not find such a flow threshold, which would probably occur at a higher flow rate.

\section{Limitations}

The present study presents several limitations. First, only one type of mechanical ventilator was used to provide all the modes of assisted ventilation compared. The results obtained with this ventilator may not be applicable to other ventilators available. However, using a single ventilator capable of providing all of the modes of assisted ventilation that the authors wanted to compare, offered the advantage of a high homogeneity of experimental conditions.

Secondly, the patient population investigated had a low breath-to-breath variability in terms of $\mathrm{RR}, V \mathrm{~T}$, and PIF at each level of selected inspiratory pressure and with relatively preserved respiratory mechanics. All patients were without COPD and were recovering from acute respiratory failure. Thus, the authors' values of WOB were relatively lower than in some other studies that investigated patients with higher ventilatory demands [31, 32].

Thirdly, setting APCV and ACV accordingly to a breathing pattern obtained during PSV facilitated the choice of comfortable setting of APCV and ACV. This is not what occurs most frequently in clinical practice, where patients are generally switched to PSV from APCV and ACV. However, the aim of the present study was to compare the three modes in the most objective way, which the authors believed they were able to accomplish with this design.

In conclusion, in a patient population with relatively preserved respiratory mechanics, it is not so important which ventilatory mode is chosen, but how each mode is regulated. The present authors identified tidal volume and peak inspiratory flow as the main parameters useful to optimising ventilatory settings. A further problem is how to find parameters, which are easily obtained at the bedside, to correctly regulate tidal volume and peak inspiratory flow for each ventilatory mode.

\section{References}

1. MacIntyre NR. Respiratory function during pressure support ventilation. Chest 1986; 89: 677-683.

2. Mathru M, Reo TL, Venes B. Ventilator induced barotrauma in controlled mechanical ventilation versus intermittent mandatory ventilation. Crit Care Med 1983; 11: 359-361.

3. Froese AB, Bryan AC. Effect of anesthesia and paralysis on diaphragmatic mechanic in man. Anesthesiology 1974; 41: 242-255.

4. Downs JB, Klein EF, Desautels D. Intermittent mandatory ventilation: a new approach to weaning patients from mechanical ventilations. Chest 1973; 64: 331-335.

5. Muller EA. Influence of training and of inactivity on muscle strength. Arch Phys Med Rehabil 1970; 51: 449-462.

6. MacIntyre NR, Ho LI. Effects of initial flow rate and breath termination criteria on pressure support ventilation. Chest 1991; 99: 134-138.

7. Brochard L, Harf A, Lorino H, Lemairie F. Inspiratory pressure support prevents diaphragmatic fatigue during weaning from mechanical ventilation. Am Rev Respir Dis 1989; 139: 513-521.

8. MacIntyre N, Nishimura M, Usada Y, Tokioka H, Takezawa J, Shimada Y. The Nagoya Conference on system design and patient ventilator interaction during pressure support ventilation. Chest 1990; 97: 14631466.

9. Amato M, Barbas C, Banasso J, Saldiva PH, Zin WA, de Carvalho R. Volume assured pressure support ventilation (VAPSV). A new approach for reducing muscle workload during acute respiratory failure. Chest 1992; 102: 1225-1234.

10. Cohen CA, Zagelbaum C, Groos D. Clinical manifestations of inspiratory muscle fatigue. $\mathrm{Am} \mathrm{J} \mathrm{Med}$ 1982; 73: 308-316.

11. Marini JJ, Rodriguez RM, Lamb V. The inspiratory workload of patient initiated mechanical ventilation. Am Rev Respir Dis 1986; 134: 902-909.

12. Marini JJ, Capps J, Culver B. The inspiratory work of breathing during assisted mechanical ventilation. Chest 1985; 87: 612-618.

13. Marini JJ, Smith T, Lamb V. External work output and force generation during synchronized intermittent mechanical ventilation. Am Rev Respir Dis 1988; 138: 1169-1179.

14. Tejeda M, Boix JH, Alvarez F, Balanza R, Morales M. Comparison of pressure support ventilation and assist control ventilation in the treatment of respiratory failure. Chest 1997; 111: 1322-1325.

15. Kreit JW, Capper MW, Eschenbacher W. Patient work of breathing during pressure support and volume-cycled mechanical ventilation. Am J Respir Crit Care Med 1994; 149: 1085-1091.

16. Baker AB, Babington PCB, Colliss JE, Cowie RW. Effect of varying inspiratory flow waveform and time in intermittent positive pressure ventilation. Introduction and methods. Br J Anaesth 1977; 49: 1207-1220.

17. Baker AB, Colliss JE, Cowie RW. Effect of varying inspiratory flow waveform and time in intermittent positive pressure ventilation. Various physiological variables. Br J Anaesth 1977; 49: 1221-1234.

18. Johansson H. Effect on breathing mechanics and gas exchange of different inspiratory gas flow patterns 
in patients undergoing respirator treatment. Acta Anaesth Scand 1975; 19: 19-27.

19. Johansson H. Effect of different inspiratory gas flow patterns on thoracic compliance during respirator treatment. Acta Anaesth Scand 1975; 19: 89-95.

20. Branson RD, Chatburn RL. New generation of microprocessor-based ventilators. In: Tobin $\mathrm{M}$, ed. Principles and Practice of Mechanical Ventilation. New York, McGraw-Hill Inc., 1994; pp. 1238-1240.

21. Pelosi $\mathrm{P}$, Cadringher $\mathrm{P}$, Bottino N, et al. Sigh in acute respiratory distress syndrome. Am J Respir Crit Care Med 1999; 159: 872-880.

22. Baydur A, Behrakis PK, Zin WA, Jaeger M, MilicEmili J. A simple method for assessing the validity of the esophageal balloon technique. Am Rev Respir Dis 1982; 126: 788-791.

23. Diehl JL, Atrouns S, Touchard D, Lemaire F, Brochard L. Changes in the work of breathing induced by tracheotomy in ventilator dependent patients. $\mathrm{Am}$ J Respir Crit Care Med 1999; 159: 383-388.

24. Brochard L, Harf A, Lorino H, Lemaire F. Inspiratory pressure support prevents diaphragmatic fatigue during weaning from mechanical ventilation. Am Rev Respir Dis 1989; 139: 513-521.

25. Murciano D, Aubier M, Bussi S, Derenne JP, Pariente R, Milic-Emili J. Comparison of esophageal pressure in patients with chronic obstructive pulmonary disease during acute respiratory failure. Am Rev Respir Dis 1982; 126: 837-841.

26. Conti G, Cinnella G, Barboni E, Lemaire F, Harf A, Brochard L. Estimation of occlusion pressure during assisted ventilation in patients with intrinsic PEEP. Am J Respir Crit Care Med 1996; 154: 907-912.

27. Tobin MJ, Yang KL, Jubran A, Lodato RF. Interrelationship of breath components in neighboring breaths of normal eupneic subjects. Am J Respir Crit Care Med 1995; 152: 1967-1976.
28. Tobin MJ, Jubran A, Laghi F. Patient ventilator interaction. Am J Respir Crit Care Med 2001; 163: 1059-1063.

29. Jubran A, Van de Graaf WB, Tobin MJ. Variability of patient-ventilator interaction with pressure support ventilation in patients with COPD. Am J Respir Crit Care Med 1995; 152: 129-136.

30. Baysen PG, McGough E. Pressure control and pressure support ventilation: Flow patterns, inspiratory time, and gas distribution. Respir Care 1988; 33: $126-134$.

31. Kallet RH, Campbell AR, Alonso JA, Marabito DJ, Mackersie RC. The effects of pressure control versus volume control assisted ventilation on patient work of breathing in acute lung injury and acute respiratory distress syndrome. Respir Care 2000; 9: 10851096.

32. Cinnella G, Conti G, Lofaso F, et al. Effects of assisted ventilation on the work of breathing: volumecontrolled versus pressure-controlled ventilation. $\mathrm{Am}$ J Respir Crit Care Med 1996; 153: 1025-1033.

33. Al Saady N, Bennett ED. Decelerating inspiratory flow waveform improves lung mechanics and gas exchange in patients on intermittent positive pressure ventilation. Intensive Care Med 1985; 11: 6875.

34. Madell HI, Cheney FW. Effects of inspiratory flow pattern on gas exchange in normal and abnormal lungs. J Appl Physiol 1979; 46: 1103-1107.

35. Ward ME, Corbeil C, Gibbons W. Optimization of respiratory muscle relaxation during mechanical ventilation. Anesthesiology 1988; 69: 29-35.

36. Chiumello D, Pelosi P, Croci M, Bigatello L, Gattinoni L. The effects of pressurization rate on breathing pattern, work of breathing, gas exchange and patient comfort in pressure support ventilation. Eur Respir J 2001; 18: 107-114. 